\title{
AN ARTIFICIAL NEURAL NETWORK APPROACH FOR THE ClASSIFICATION OF HuMAN LOWER BACK PAIN
}

\author{
Shubham Sharma and Rene V.Mayorga \\ Industrial Systems Engineering, University of Regina, Canada
}

\begin{abstract}
In today's world, the problem of lower back pain is one of the fastest growing crucial ailments to deal with. More than half of total population on the earth, suffers from it at least once in a lifetime. Human Lower Back Pain symptoms are commonly categorized as Normal or Abnormal. In order to remedy Human Lower Back Pain, with the growth of technology over the time, many medical methods have been developed to diagnose and cure this pain at its earliest stage possible. This study aims to develop two Machine Learning (M.L.) models which can classify Human Lower Back Pain symptoms in a human body using non-conventional techniques such as Feedforward/Backpropagation Artificial Neural Networks, and Fully Connected Deep Networks. An Automatic Feature Engineering technique is implemented to extract featured data used for the classification. The proposed models are compared with respect to a Support Vector Machine model; considering different performance parameters.
\end{abstract}

\section{KEYWORDS}

Machine Learning, Artificial Neural Networks, Fully Connected Deep Networks, Support Vector Machine, Lower Back Pain, Automatic Feature Engineering technique.

\section{INTRODUCTION}

According to statistics, near about $80 \%$ of adults go through the pain of lower back at some point in their life [1]. Although there has been a noted increase in the technologies and the number of chiropractors to deal with lower back pain; still the ratio of the LBP patients and the chiropractors is quite large. The parts of back involved in this pain are mainly an arrangement of spine, spinal cord, the disc like structure between vertebrates and the ligaments which connects bone to bone.

Lin L. et al [2006], published work named "A Decision Support System for lower back pain diagnosis: uncertainty management and clinical evaluations". This system is a typical web-based system where all the verification and system validation were done using Turing test [2]. Fourney D. et al [2011], presented a review of clinical pathways for lower back pain and case study of the Saskatchewan Spine Pathway. The main motto of this research was to find differences between clinical pathways and clinical guidelines, its example and testing of its success and about SSP [3]. Jenkins H. [2002], presented a paper in which he mentioned about the classification of low back pain. It described about the different types of lower back pain and its classification using KNN, Logistic Regression, Naïve Bayes, Random Forest, Decision Tree, and CART [4].

Natarajan Meghanathan et al. (Eds) : CSEIT, CMLA, NeTCOM, CIoT, SPM, NCS, WiMoNe, Graph-hoc - 2019 pp. 167-172, 2019. (C) CS \& IT-CSCP 2019

DOI: $10.5121 /$ csit.2019.91313 
In this paper, the Classification Models are generated using different Machine Learning techniques such as: The Support Vector Machine (SVM) method; the Feedforward/Backpropagation Artificial Neural Networks (ANN) technique; and the Fully Connected Deep Network Algorithm, (FCDNA), [5][6][7]. Training and testing of the above classification models are done using a publicly available dataset [8]. This dataset consists of 13 columns, from which the first 12 columns are commonly termed as pelvic parameters or Range of Motion (ROM) Attributes. The Final column of the dataset indicates whether the first 12 column values or pelvic parameters values are Normal back pain or Abnormal back pain symptoms. Range of Motion (ROM) Attributes contained in the dataset are named as follows:

Attribute Label Attribute Name

Table 1. Range of Motion (ROM) Attributes

\begin{tabular}{|c|l|}
\hline Attribute Label & Attribute Name \\
\hline Coll & Pelvic Incidence \\
\hline Col2 & Pelvic Tilt \\
\hline Col3 & $\begin{array}{l}\text { Lumbar Lordosis } \\
\text { Angle }\end{array}$ \\
\hline Col4 & Sacral Slope \\
\hline Col5 & Pelvic Radius \\
\hline Col6 & $\begin{array}{l}\text { Degree } \\
\text { Spondylolisthesis }\end{array}$ \\
\hline Col7 & Pelvic Slope \\
\hline Col3 & Direct Tilt \\
\hline Coll0 & Thoracic Slope \\
\hline Coll1 & Servical Tilt \\
\hline Coll2 & Sacrum Angle \\
\hline & Scoliosis Slope \\
\hline
\end{tabular}

This paper explains the application of various Machine Learning techniques to correctly classify lower back pain symptoms using featured data obtained by implementing the Automatic Feature Engineering technique on the complete dataset. Section 2 explains the methodology which includes data preprocessing, model generation, and performance analysis. Section 3 explains the results after comparing various models and Section 4 presents some Conclusions.

\section{Methodology}

\subsection{Data Preprocessing}

Original dataset is retrieved from a website named Kaggle [8]. Firstly, the dataset normalized and reordered in Waikato Environment [9]. Automatic Feature Engineering technique is applied on the normalized to extract the features. Preprocessing of data can be shown as below.

Featured columns for this dataset after data reduction process are [col1], [col2], [col3], [col4], [col5], [col5/col10] [col6], sqrt[col5/col10]. These featured data columns are the inputs to the classification models. 


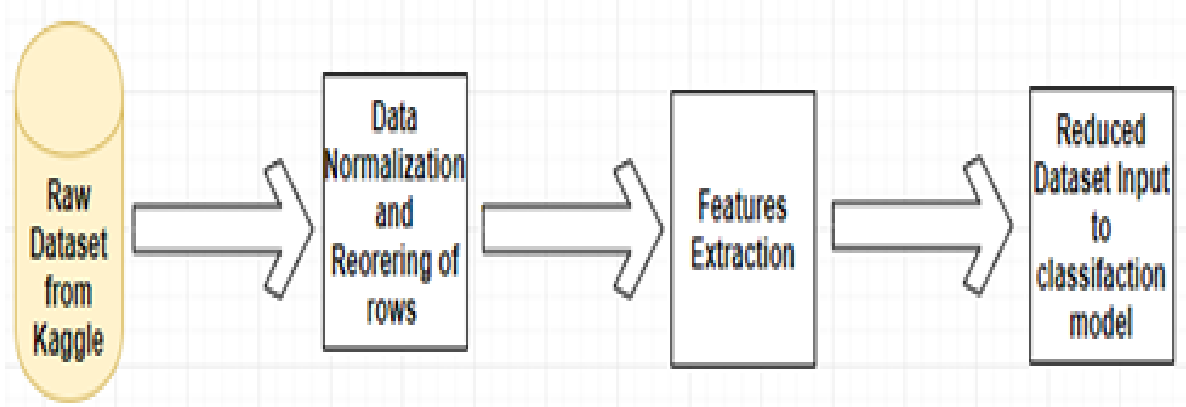

Fig. 1 Data Collection and Preprocessing

\subsection{Model Building}

Three different classification models are built in this research. Simulation of these models are performed in Rapid Miner Software [10]. This software provides a Graphical User Interface (GUI) for the analytical workflows or commonly termed as process. A process consists of a combination of different operators where each operator is required to do a specific task [10].

The Proposed Artificial Neural Network Classification Model has 2 hidden layers having 8 and 5 neurons in layer 1 and layer 2 respectively. The Activation function is Rectifier and Number of epochs are 100. Stochastic gradient descent (SGD) method is used here to minimize the loss function [11].

The Deep learning Classification Model designed in this research consists of three fully connected hidden layers containing 18, 80 and 2 neurons in layer 1,2 and 3 respectively; with Activation function RelU (Rectifier Linear Unit) in first 2 and SoftMax in last layer [12][13]. The SGD method is used here to minimize the loss function.

The Support Vector Machine model is using C-SVC type SVM structure, Kernel type: Rbf, Gamma Value: 1.0000000000007, C value:100 and epsilon:0.001 [14].

\subsection{Training and Testing}

The dataset consists of 311 datapoints out of which 187 datapoints (60\% of dataset) are used for training of the classifiers and rest 124 datapoints (40\% of dataset) are used for the testing of the trained models and the performance of the models is compared on the basis of performance parameters defined in next section.

\subsection{Performance Evaluation Parameters}

Performance of the proposed Machine Learning based Classification models are evaluated on the parameters such as Accuracy, Precision, F-measure, Sensitivity, Specificity and Area under Curve (AUC) whose formulae are listed below.

$$
\begin{gathered}
\text { Accuracy }=(\mathrm{TP}+\mathrm{TN}) /(\mathrm{TP}+\mathrm{TN}+\mathrm{FP}+\mathrm{FN}) \\
\text { Precision }=\mathrm{TP} /(\mathrm{TP}+\mathrm{FP}) \\
\text { Recall (or Sensitivity) }=\mathrm{TP} /(\mathrm{TP}+\mathrm{FN})
\end{gathered}
$$




$$
\begin{gathered}
\text { Specificity }=\mathrm{TN} /(\mathrm{TN}+\mathrm{FP}) \\
\text { F-measure }=2 * \text { Precision*Recall } /(\text { Precision }+ \text { Recall })
\end{gathered}
$$

[TN- True Negative, TP- True Positive, FN- False Negative, FP- False Positive] ..[15].

AUC is used to know which model predicts the best classification. In this curve, Positive Instances are plotted against negative instances and results are compared [16].

\section{RESUltS AND COMPARISON}

All the above proposed models are designed, and comparison results are shown in table below.

Table 2. Performance Comparison

\begin{tabular}{|c|c|c|c|c|c|c|c|}
\hline $\begin{array}{c}\text { S. } \\
\text { No. }\end{array}$ & $\begin{array}{c}\text { Classification } \\
\text { Model }\end{array}$ & $\begin{array}{c}\text { Accuracy } \\
\text { (in \%) }\end{array}$ & $\begin{array}{c}\text { Precision } \\
\text { (in \%) }\end{array}$ & $\begin{array}{c}\text { F- } \\
\text { measure } \\
\text { (in \%) }\end{array}$ & $\begin{array}{c}\text { Specificity } \\
\text { (in \%) }\end{array}$ & $\begin{array}{c}\text { Sensitivity } \\
\text { (in \%) }\end{array}$ & $\begin{array}{c}\text { AUC } \\
\text { (in \%) }\end{array}$ \\
\hline 1 & ANN & 88.6 & 90.3 & 91.8 & 78.6 & 93.3 & 90.1 \\
\hline 2 & $\begin{array}{c}\text { Deep } \\
\text { Network }\end{array}$ & 83.9 & 88.1 & 88.1 & 75 & 88 & 92.9 \\
\hline 3 & SVM & 80.7 & 86.9 & 85.7 & 69.8 & 84.8 & 86.9 \\
\hline
\end{tabular}

The Classification models are compared based on six performance parameters. As clear from table 2, the ANN classification Model gives best accuracy (88.6\%) among all, and the Deep Learning model is second best (83.9\%). In terms of precision, the ANN gives best result (90.3\%) and the Deep Learning model comes after that (88.1\%). The ANN is the best model among the three models in terms of Sensitivity and Specificity with a value of $93.3 \%$ and $78.6 \%$ for respective parameter. In terms of Area Under Curve (AUC), the proposed Deep Learning model gives best figure $(92.9 \%)$ and the ANN is second best in this case (90.1\%).

The computational time for each developed model is depicted in following table.

Table 3. Computational Times

\begin{tabular}{|c|c|c|}
\hline S. No. & Classification Model & Computational Time (in Sec) \\
\hline 1 & ANN & 7 \\
\hline 2 & Deep Network & 7 \\
\hline 3 & SVM & 4 \\
\hline
\end{tabular}

Weights of attributes for the featured inputs are shown in the following table (Table 4) with respect to each classification model. Weight of an attribute signifies the impact of that input in the classification of the data. 
Table 4. Weights of Attributes

\begin{tabular}{|c|c|c|c|c|}
\hline \multicolumn{2}{|c|}{ S. No. } & Featured Input Data & \multicolumn{3}{|c|}{ Classification Model } \\
\hline \multicolumn{1}{|c|}{} & Col1 & ANN & Deep Learning & SVM \\
\hline 1 & Col2 & 0.017 & 0.449 & 0.026 \\
\hline 2 & Col3 & 0.028 & 0.089 & 0.025 \\
\hline 3 & Col4 & 0.067 & 0.027 & 0.035 \\
\hline 4 & Col5 & 0.041 & 0.063 & 0.019 \\
\hline 5 & Col5/Col10 & 0.055 & 0.082 & 0.012 \\
\hline 6 & Col6 & 0.45 & 0.449 & 0.006 \\
\hline 7 & sqrt(Col5/Col10) & 0.039 & 0.007 & 0.106 \\
\hline 8 & & & & 0.037 \\
\hline
\end{tabular}

It is interesting to notice the somewhat unexpected results given by the Fully Connected Deep Network model based on the test data. It appears that the performance results are not satisfactory enough due to an insufficient amount of data to train this model. The Deep Learning model can perform well when the amount of dataset is large to train the model. The SVM algorithm-based classification model does not perform that well either; due to the limitation of the framework used for the simulation. This study successfully classifies Human Lower Back Pain data using different classification models. It is observed that the highest accuracy $88.64 \%$ is yielded by ANN model; whereas, the lowest accuracy of $80.7 \%$ is obtained from the SVM model.

\section{CONCLUSIONS}

This paper provides a non-conventional approach to detect lower back pain in a human body. Twelve Range of Motion attributes' values can decide the type of lower back pain; which is either Normal or Abnormal. Some Machine Learning techniques such as: ANN, Deep learning, and the SVM are used to generate classification models which give promising results. The ANN model gives the best results in terms of Accuracy, Precision, F-measure, Sensitivity and Specificity; while the Deep Learning model is the best model in terms of AUC. Hence, for the practical use, it can be concluded that the proposed the ANN based Classification model can be considered as the "best" model amongthe three models. Therefore, the ANN model can be used as Clinical Decision Support System (CDSS) by the physicians or chiropractors. The Deep learning model's lower accuracy can be explained by the low amount of available data used in this study. Future work can be done in this field to increase the performance of classification models. Recommendation includes large dataset to train and test the models, trying different algorithms with fine tuning, and other different frameworks to implement this study.

\section{ACKNOWLEDGEMENT}

This paper research has been supported by a grant from the Natural Sciences and Engineering Research Council of Canada (NSERC).

\section{REFERENCES}

[1] Rubin, D. I. (2007). Epidemiology and risk factors for spine pain. Neurologic clinics, 25(2), 353-371. 
[2] Lin, Lin \& Jen-Hwa Hu, Paul \& Sheng, Olivia. (2006). A decision support system for lower back pain diagnosis: Uncertainty management and clinical evaluations. Decision Support Systems. 42. 11521169. 10.1016/j.dss.2005.10.007.

[3] Fourney, D. R., Dettori, J. R., Hall, H., Härtl, R., McGirt, M. J., \& Daubs, M. D. (2011). A systematic review of clinical pathways for lower back pain and introduction of the Saskatchewan Spine Pathway. Spine, 36, S164-S171.

[4] Jenkins, H. (2002).Classification of low back pain. Australasian Chiropractic \& Osteopathy,10(2), 91.

[5] Lau, Suki (10 July 2017). "A Walkthrough of Convolutional Neural Network - Hyperparameter Tuning". Medium. Retrieved 23 August 2019.

[6] TensorFlow for Deep Learning. (n.d.). Retrieved August 6, 2019, from <https://learning.oreilly.com/library/view/tensorflow-for-deep/9781491980446/ch04.html>.

[7] Cortes, Corinna; Vapnik, Vladimir N. (1995). "Support-vector networks". Machine Learning. 20 (3): 273-297. CiteSeerX 10.1.1.15.9362. doi:10.1007/BF00994018.

[8] Lower Back Pain symptoms dataset, Version 1. Retrieved on May 17, 2019 from <https://www.kaggle.com/sammy123/lower-back-pain-symptoms-dataset>.

[9] Eibe Frank, Mark A. Hall, and Ian H. Witten (2016). The WEKA Workbench. Online Appendix for "Data Mining: Practical Machine Learning Tools and Techniques", Morgan Kaufmann, Fourth Edition.

[10] GmbH, R. (n.d.). Fast Large Margin (RapidMiner Studio Core). Retrieved July 3, 2019, from $<$ https://docs.rapidminer.com/latest/studio/operators/modeling/predictive/support_vector_machines/fa st_large_margin.html>.

[11] Taddy, Matt (2019). "Stochastic Gradient Descent". Business Data Science: Combining Machine Learning and Economics to Optimize, Automate, and Accelerate Business Decisions. New York: McGraw-Hill. pp. 303-307. ISBN 978-1-260-45277-8.

[12] Brownlee, J. (2019 7). A Gentle Introduction to the Rectified Linear Unit (ReLU). Retrieved 27, 2019, from https://machinelearningmastery.com/rectified-linear-activation-function-for-deeplearning-neural-networks/

[13] Goodfellow, Ian; Bengio, Yoshua; Courville, Aaron (2016). "6.2.2.3 Softmax Units for Multinoulli Output Distributions". Deep Learning. MIT Press. pp. 180-184. ISBN 978-0-26203561-3.

[14] RBF SVM parameters. (n.d.). Retrieved August 27, 2019, from <https://scikitlearn.org/stable/auto_examples/svm/plot_rbf_parameters.html>.

[15] Sunasra, M. (2019, February 28). Performance Metrics for Classification problems in Machine Learning. Retrieved August 8, 2019, from <https://medium.com/thalus-ai/performance-metrics-forclassification-problems-in-machine-learning-part-i-b085d432082b>.

[16] Classification: ROC Curve and AUC | Machine Learning Crash Course. (n.d.). Retrieved September 8, 2019, from <https://developers.google.com/machine-learning/crash-course/classification/roc-andauc>. 